\title{
Small-Angle Shubnikov-de Haas Measurements in a 2D Electron System: The Effect of a Strong In-Plane Magnetic Field
}

\author{
Sergey A. Vitkalov, Hairong Zheng, K. M. Mertes, and M. P. Sarachik \\ Physics Department, City College of the City University of New York, New York, New York 10031 \\ T. M. Klapwijk \\ Delft University of Technology, Department of Applied Physics, 2628 CJ Delft, The Netherlands
}

(Received 13 April 2000)

\begin{abstract}
Measurements in magnetic fields applied at small angles relative to the electron plane in silicon MOSFETs indicate a factor of 2 increase of the frequency of Shubnikov-de Haas oscillations at $H>H_{\text {sat }}$. This signals the onset of full spin polarization above $H_{\text {sat }}$, the parallel field above which the resistivity saturates to a constant value. For $H<H_{\text {sat }}$, the phase of the second harmonic of the oscillations relative to the first is consistent with scattering events that depend on the overlap instead of the sum of the spin-up and spin-down densities of states. This unusual behavior may reflect the importance of many-body interactions.
\end{abstract}

PACS numbers: 71.30. $+\mathrm{h}, 72.20 . \mathrm{My}, 73.40 . \mathrm{Hm}, 73.40 . \mathrm{Qv}$

A great deal of interest has recently been focused on the anomalous behavior of two-dimensional (2D) systems of electrons [1,2] and holes [3-5] whose resistivities unexpectedly decrease with decreasing temperature, behavior that is generally associated with metals rather than insulators [6]. One of the most intriguing characteristics of these systems is their enormous response to magnetic fields applied in the plane of the electrons [7-9] or holes [5,10]: the resistivity increases sharply by more than an order of magnitude, saturating to a constant plateau value above a magnetic field $H_{\text {sat }}$.

In this paper we report studies of the resistivity of the 2D electron system in silicon metal-oxide-semiconductor field-effect transistors (MOSFETs) in magnetic fields applied at small angles $\phi$ with respect to the plane. This allows a study of the Shubnikov-de Haas ( $\mathrm{SdH})$ oscillations in perpendicular fields sufficiently small that the orbital motion has a negligible effect on the response to the in-plane component of the magnetic field. At small tilt angles $\phi$, the $\mathrm{SdH}$ oscillations plotted versus filling factor have twice the period below $H_{\text {sat }}$ compared with the period above $H_{\text {sat }}$. This implies that the electron system is fully spin polarized in high fields, $H>H_{\text {sat }}$, where the resistivity has reached saturation. Detailed examination of the oscillations in fields below $H_{\text {sat }}$ indicates unusual behavior consistent with electron scattering that depends on the product rather than the sum of the spin-up and spin-down densities of states.

Two silicon MOSFETs with mobilities $\mu \approx$ $20000 \mathrm{~V} / \mathrm{cm}^{2} \mathrm{~s}$ at $T=4.2 \mathrm{~K}$ were used in these studies. Contact resistances were minimized by using samples with a split-gate geometry, which permit high densities in the vicinity of the contacts while allowing independent control of the density of the $2 \mathrm{D}$ system under investigation. Standard $A C$ four-probe techniques were used at $3 \mathrm{~Hz}$ to measure the resistance in the linear regime using currents typically below $5 \mathrm{nA}$. Data were taken on samples mounted on a rotating platform in a ${ }^{3} \mathrm{He}$ Oxford Heliox system at temperatures down to $0.235 \mathrm{~K}$ in magnetic fields up to $12 \mathrm{~T}$.

Measurements were first taken with the plane of the sample oriented parallel to the magnetic field [11]. The resistance, $R_{x x}$, is shown in Fig. 1 as a function of field for different fixed gate voltages spanning densities between $n_{s}=0.8 \times 10^{11} \mathrm{~cm}^{-2}$ and $n_{s}=2.88 \times 10^{12} \mathrm{~cm}^{-2}$ (the zero field critical density for the metal-insulator transition is $n_{c} \approx 0.84 \times 10^{11} \mathrm{~cm}^{-2}$ ). Consistent with earlier findings $[7,8,10,12,13]$, the in-plane $(\phi=0)$ magnetoresistance rises dramatically with increasing field and saturates above a density-dependent field $H_{\text {sat }}\left(n_{s}\right)$ [14].

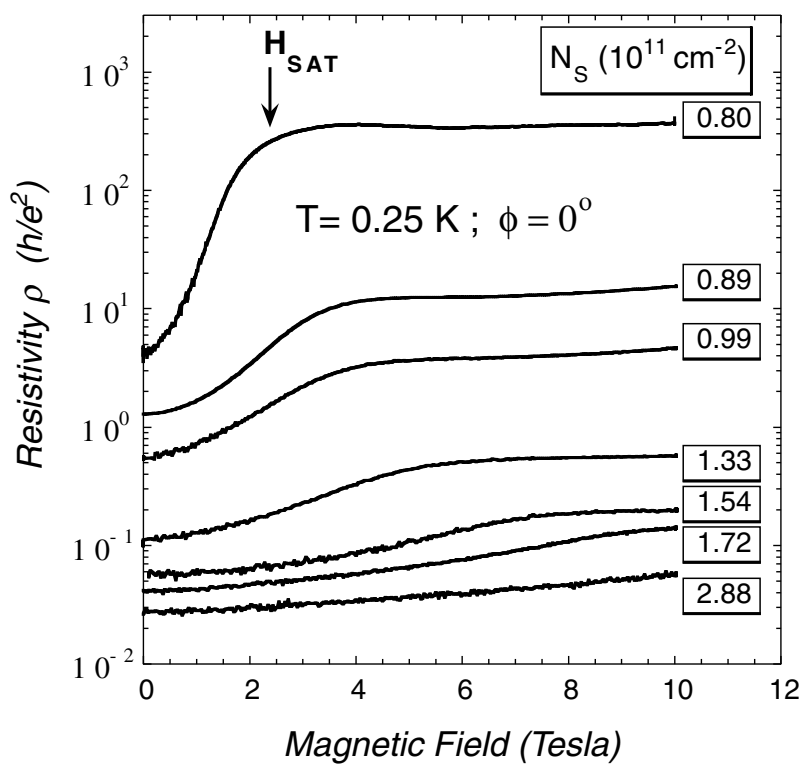

FIG. 1. Resistivity of the 2D electron system in silicon MOSFETs versus in-plane magnetic field for different densities, as labeled. Data are shown at $T=245 \mathrm{mK}$. The arrow indicates $H_{\text {sat }}$ for electron density $n_{s}=0.80 \times 10^{11} \mathrm{~cm}^{-2}$. 
The sample was then rotated to make a small angle $\phi$ with respect to the field, so that the in-plane component was almost equal to the total field $H_{\|} \approx H$, while the projection in the perpendicular direction, $H_{\perp} \approx \phi H$, remained relatively small even in high fields. $R_{x x}$ and $R_{x y}$ were measured simultaneously as a function of magnetic field for fixed angle $\phi$, temperature $T$, and density $n_{s}$.

For various different densities $n_{s}$, Figs. 2(a) and 2(b) show the resistance $R_{x x}$ vs filling factor $\nu$ at two different angles $\phi$ between the magnetic field and the 2D plane. Similar curves were obtained at other small angles. The filling factor $\nu=n_{s} \Phi_{0} / H_{\perp}$ was calculated using the relation $n_{s}=H_{\perp} /\left(R_{x y} e c\right)$. The Hall resistivity $R_{x y}$ and Hall coefficient $R_{H}$ were determined from the low-field data, $R_{x y}=R_{H} \times H_{\perp}$, (i.e., in fields below the onset of quantum oscillations).

For the lowest densities shown in Fig. 2(a) at angle $\phi=6^{\circ}$, the system is in the high-field saturated regime above $H_{\text {sat }}$ for filling factor $\nu<10$. The large arrow indicates $H_{\text {sat }}$ for $n_{s}=1.54 \times 10^{11} \mathrm{~cm}^{-2}$. Quantum oscillations are clearly evident superimposed on the large plateau

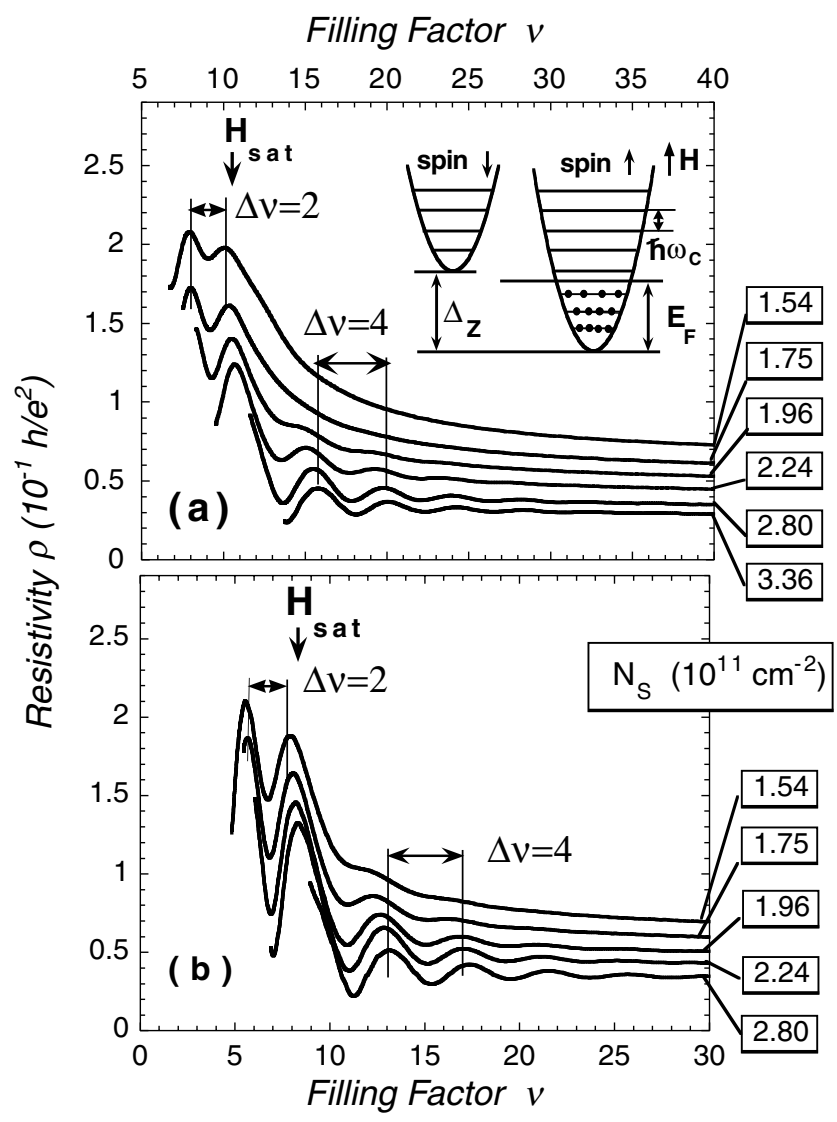

FIG. 2. Resistivity $\rho_{x x}$ versus electron filling factor $\nu=$ $n_{s} \Phi_{0} / H_{\perp}$ showing $\mathrm{SdH}$ oscillations for different electron densities $n_{s}$, as labeled. Data are shown at $T=0.25 \mathrm{~K}$ for two different angles $\phi$ between the magnetic field and the electron plane: (a) $\phi=6^{\circ}$; (b) $\phi=7.8^{\circ}$. Note that $H_{\perp}$ increases to the left. value of $R_{x x}$ at small $\nu$. In this region the period of the $\mathrm{SdH}$ oscillations corresponds to a change in filling factor $\Delta \nu_{\text {sat }}=2$ [including the twofold valley degeneracy for a 2D layer of (100) silicon]. For higher filling factors $\nu>10$ where the 2D electron system is below saturation $\left(H<H_{\text {sat }}\right)$, the period of the $\mathrm{SdH}$ oscillations is twice as long, namely, $\Delta \nu=4$. Similar behavior is shown for a bigger angle in Fig. 2(b), where the larger perpendicular component gives rise to stronger $\mathrm{SdH}$ oscillations; here the period doubling is found above $\nu \approx 8$.

The period $\Delta \nu=2$ of the oscillations at $H>H_{\text {sat }}$ corresponds to complete spin polarization of the electrons. A number of interesting suggestions have been advanced to account for the unusual behavior of these systems, including superconductivity, a Wigner crystal or glass, various esoteric non-Fermi liquid scenarios, as well as ordinary Fermi liquid behavior [6]. Here we restrict the discussion to a simple model within a single particle description. Using this approach we were able to explain the doubling of the frequency of the SdH oscillations at $H>H_{\text {sat }}$. However, the detailed behavior of the oscillations in small perpendicular fields is not fully consistent with this model.

As shown schematically in the inset of Fig. 2, the spin-up and spin-down electron bands are split by the Zeeman energy $\Delta_{Z}=g \mu_{B} H$, while the spacing between the Landau levels, $\hbar \omega_{c}=\hbar e H_{\perp} / m c$, is determined by the perpendicular component of the field. We consider the progression as electrons are added to the system: for small densities, $E_{F}<\Delta_{Z}$, electrons are added to Landau levels in the spin-up band only, corresponding to a $\mathrm{SdH}$ periodicity $\Delta \nu=2$ (including a factor of 2 for the valley degeneracy in silicon); at high densities, $E_{F}>\Delta_{Z}$, twice as many electrons are required to fill both spin-up and spin-down Landau levels, yielding the double period, or $\Delta \nu=4$ [15]. An equivalent argument holds for fixed density as one reduces the magnetic field. Thus, the shorter period $\Delta \nu=2$ at $H>H_{\text {sat }}$ signals the onset of full polarization of the electron system [16]. The relationship between $H_{\text {sat }}$ and complete spin polarization was also found by Okamoto et al. [13] using a different experimental method.

Clearly, the observed period doubling is consistent with this simple model only if the spin-up and spin-down levels are degenerate or nearly so, so that $\alpha=\Delta_{Z} / \hbar \omega_{c}=i$ with $i$ an integer. The double period should revert to a single period when $\alpha=i+1 / 2$, corresponding to a spin-up Landau level between two spin-down Landau levels. The ratio $\alpha$ can be varied experimentally by changing the angle $\phi$, or by using the fact that the electron-electron interaction-enhanced $g$ factor (and thus $\Delta_{Z}$ ) decreases with increasing electron density in silicon MOSFETs $[13,17]$. By taking data over a broad range of densities, we were able to smoothly vary $\alpha$ by more than 1. Close examination of the data shows that the double period in fields below $H_{\text {sat }}$, although stable over a broad range, does break down in a narrow range of densities that 
is different for different angles $\phi$, as will be discussed within this model for the data of Fig. 3.

$\mathrm{SdH}$ oscillations reflect changes in electron scattering due to periodic oscillations of the density of states at the Fermi level [18]. For the weak perpendicular fields used in our experiments, there is strong scattering and the $\mathrm{SdH}$ oscillations are small [19]. Unlike the situation that prevails in high magnetic fields, where the Landau levels are sharp and well defined, the density of states is best represented in this regime by a harmonic expansion [17]:

$$
\begin{gathered}
D_{\downarrow, \uparrow}(E)=D_{0}\left\{1+\epsilon \times \cos \left[2 \pi\left(E \pm \Delta_{Z} / 2\right) / \hbar \omega_{c}\right]\right. \\
\left.+O\left(\epsilon^{2}\right)\right\} .
\end{gathered}
$$

Here $E, \Delta_{Z}=g \mu_{B} H$, and $\hbar \omega_{c}$ are the energy, Zeeman energy, and cyclotron energy, respectively. The small parameter $\epsilon=2 \exp \left[-\pi /\left(\omega_{c} \tau\right)\right] \ll 1$ [17] is proportional to the Dingle factor [19]. Small variations in the resistivity are proportional to small variations in the scattering probability $W: \Delta \rho / \rho=\Delta W / W$. Using the Born approximation, $W \sim \int \delta\left(E-E_{F}\right) D(E) d E$, one can show that

$$
\Delta \rho / \rho= \begin{cases}\epsilon \times \cos \left(\pi \Delta_{Z} / \hbar \omega_{c}\right) \cos \left(2 \pi E_{F}^{0} / \hbar \omega_{c}\right), & \Delta_{Z}<2 E_{F}^{0}, \\ \epsilon \times \cos \left(4 \pi E_{F}^{0} / \hbar \omega_{c}\right), & \Delta_{Z}>2 E_{F}^{0} .\end{cases}
$$

Here the Fermi energy $E_{F}$ is measured from the bottom of the band at $H=0, E_{F}^{0}$ is the Fermi energy at $H=0$, and $D(E)$ is the total density of states: $D(E)=$ $D_{\uparrow}(E)+D_{\downarrow}(E)$. We assumed $T=0$ and neglected higher harmonic terms of order $\epsilon^{2}$ in Eq. (1) as well as higher order corrections due to oscillations of the Fermi energy. This demonstrates that the $\mathrm{SdH}$ period changes by a factor of 2 when $\Delta_{Z}>2 E_{F}^{0}$, corresponding to full polarization of the electrons.

The term $A=\epsilon \cos \left(\pi \Delta_{Z} / \hbar \omega_{c}\right)$ of Eq. (2) depends on the ratio $\alpha=\Delta_{Z} / \hbar \omega_{c}$, which is fixed for a given angle and electron density and does not vary with magnetic field. It determines the overall amplitude of the oscillations at fields below $H_{\text {sat }}$, when $\Delta_{Z}<2 E_{F}^{0}$. This amplitude has a maximum when $\alpha=\Delta_{Z} / \hbar \omega_{c}=i$ is an integer, corresponding to spin-up and spin-down densities of states oscillating in phase, and vanishes when $\alpha=i+1 / 2$ [see Eq. (1)]. Figure 3 shows data over a narrow region near $\alpha=i+1 / 2$ where the amplitude of the first harmonic

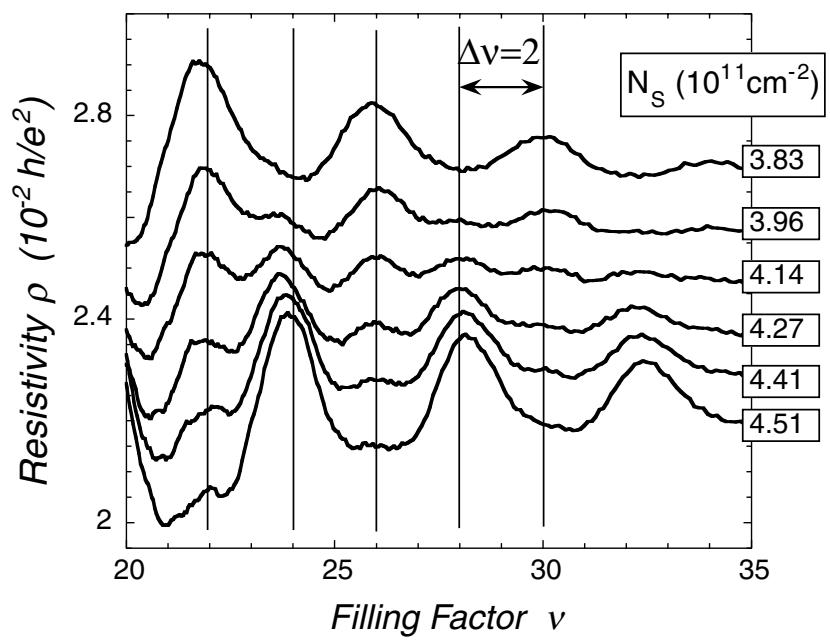

FIG. 3. In magnetic fields below saturation, $H<H_{\text {sat }}$, the $\mathrm{SdH}$ oscillations are shown for a narrow range of electron densities near $\alpha=\Delta_{Z} / \hbar \omega_{c}=g \mu_{B} H / \hbar \omega_{c}=(i+1 / 2)$; due to the $e$-e interaction enhancement of the $g$ factor, each density corresponds to a slightly different value of $\alpha$. Note that the maxima of the second harmonic $\left(n_{s}=4.14 \times 10^{11} \mathrm{~cm}^{-2}\right)$ are in phase with the maxima of the first harmonic $\left(n_{s}=3.83\right.$ and $\left.4.51 \times 10^{11} \mathrm{~cm}^{-2}\right)$. is small, allowing detailed examination of the next higher harmonic term [see Eq. (1)] with period $\Delta \nu=2$.

Based on the usual assumption that the $\mathrm{SdH}$ oscillations are determined by the total density of states, $D(E)=D_{\uparrow}(E)+D_{\downarrow}(E)$, one expects and generally observes [20] the progression illustrated schematically in Figs. 4(a)-4(c): a minimum which becomes progressively deeper develops at the center of each maximum [see curves $4(a)$ and $4(b)$ ], gradually splitting it into two separate maxima [curve 4(c)]. Thus, the minima of the second harmonic [curve $4(c)$ ] are at the positions of the maxima of the first harmonic [curve $(a)$ in Fig. 4].

However, careful examination of the data of Fig. 3 shows that the behavior below $H_{\text {sat }}$ observed experimentally in silicon MOSFETs is quite different: no minima develop within the maxima, splitting them into two;

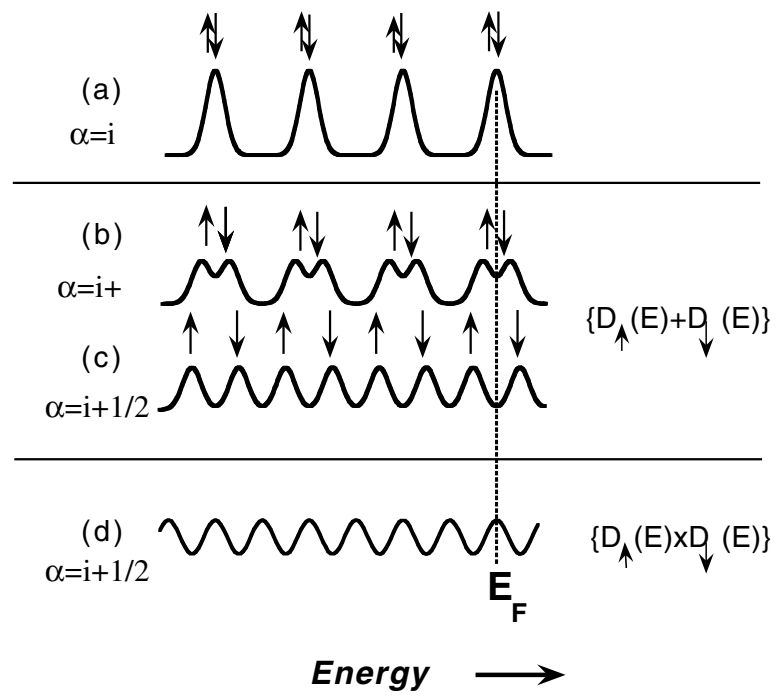

FIG. 4. Schematic of SdH oscillations for a density of states $D(E)=D_{\uparrow}(E)+D_{\downarrow}(E)$ when $(a)$ the spin-up and spin-down Landau levels are degenerate, $\alpha=\Delta_{Z} / \hbar \omega_{c}=i ;(b) \alpha=i+$ $\delta$ for small $\delta$; and (c) $\alpha=i+1 / 2$. Note that the maxima in $(a)$ and $(c)$ are $180^{\circ}$ out of phase. Curve $(d)$ illustrates maxima for the second harmonic in phase with maxima of the first obtained from assuming a scattering probability of the form $W=f\left(D_{\uparrow}(E) \times D_{\downarrow}(E)\right)$, in agreement with the experimental behavior shown in Fig. 3 . 
instead, the maxima simply diminish in amplitude and new neighboring maxima appear and grow in amplitude. The maxima of the first $\left(n_{s}=3.83\right.$ and $\left.4.51 \times 10^{11} \mathrm{~cm}^{-2}\right)$ and second $\left(n_{s}=4.14 \times 10^{11} \mathrm{~cm}^{-2}\right)$ harmonics are in phase [as in curves $(a)$ and $(d)$ of Fig. 4] rather than $180^{\circ}$ out of phase. The origin of this unusual behavior is not clear and warrants further careful study. Interestingly, the phase relation between first and second harmonics observed in our experiments can be obtained within the single particle model used earlier if one considers the product of spin-up and spin-down densities of states, $W=f\left(D_{\uparrow}(E) \times D_{\downarrow}(E)\right)$, rather than their sum; curve $(d)$ of Fig. 4 is the result of such a calculation. This suggests there is a sizable contribution to the electron scattering from events that depend on the overlap of spin-up and spin-down densities of states, perhaps reflecting enhanced scattering of electrons of opposite spin.

In summary, measurements of small-angle Shubnikovde Haas oscillations indicate that the period of the oscillations changes by a factor of 2 at the magnetic field $H_{\text {sat }}$ above which the resistance has reached saturation. We attribute the abrupt change in period to the onset of full polarization of the electron spins. The period doubling in fields below $H_{\text {sat }}$ is stable with respect to the angle between the magnetic field and the 2D plane, and is observed for all electron densities except in a narrow interval, where the amplitude of the first harmonic of the SdH oscillations vanishes and the second harmonic is observable. The phase observed for the second harmonic relative to the first is consistent with $\mathrm{SdH}$ oscillations due to scattering events that depend on the overlap instead of the sum of the spin-up and spin-down densities of states at the Fermi level. This unusual behavior may reflect the importance of many-body interactions in the $2 \mathrm{D}$ system.

We are grateful to S. Bakker and R. Heemskerk for their contributions in developing and preparing the MOSFETs used in this work. We thank E. Abrahams, L. Ioffe, F. Fang, A. Fowler, S. V. Kravchenko, A. Shashkin, X. Si, S. Chakravarty, D. Schmeltzer, F. Stern, M. Raikh, and U. Lyanda-Geller for illuminating discussions. We are grateful to A. Shashkin and S. V. Kravchenko for valuable comments on the manuscript. This work was supported by the U.S. Department of Energy Grant No. DE-FG0284ER45153.

[1] S. V. Kravchenko, G. V. Kravchenko, J.E. Furneaux, V. M. Pudalov, and M. D'Iorio, Phys. Rev. B 50, 8039
(1994); S. V. Kravchenko, W.E. Mason, G. E. Bowker, J. E. Furneaux, V. M. Pudalov, and M. D'Iorio, Phys. Rev. B 51, 7038 (1995); S. V. Kravchenko, D. Simonian, M. P. Sarachik, Whitney Mason, and J. Furneaux, Phys. Rev. Lett. 77, 4938 (1996).

[2] D. Popović, A. B. Fowler, and S. Washburn, Phys. Rev. Lett. 79, 1543 (1997).

[3] P. T. Coleridge, R. L. Williams, Y. Feng, and P. Zawadzki, Phys. Rev. B 56, R12 764 (1997).

[4] Y. Hanein, U. Meirav, D. Shahar, C. C. Li, D. C. Tsui, and Hadas Shtrikman, Phys. Rev. Lett. 80, 1288 (1998).

[5] M. Y. Simmons, A. R. Hamilton, M. Pepper, E. H. Linfield, P. D. Rose, D. A. Ritchie, A. K. Savchenko, and T. G. Griffiths, Phys. Rev. Lett. 80, 1292 (1998).

[6] For a review, see E. Abrahams, S. V. Kravchenko, and M. P. Sarachik, cond-mat/0006055; M.P. Sarachik and S. V. Kravchenko, Proc. Natl. Acad. Sci. U.S.A. 96, 5900 (1999).

[7] D. Simonian, S. V. Kravchenko, M. P. Sarachik, and V. M. Pudalov, Phys. Rev. Lett. 79, 2304 (1997).

[8] V.M. Pudalov, G. Brunthaler, A. Prinz, and G. Bauer, Pis'ma Zh. Eksp. Teor. Fiz. 65, 887 (1997) [JETP Lett. 65, 932 (1997)].

[9] V. Dolgopolov, G. V. Kravchenko, A. A. Shashkin, and S. V. Kravchenko, JETP Lett. 55, 733 (1992).

[10] J. Yoon, C. C. Li, D. Shahar, D. C. Tsui, and M. Shayegan, cond-mat/9907128.

[11] Parallel alignment was determined by choosing the angle for which $R_{x y}=0$.

[12] K. Mertes, D. Simonian, M. P. Sarachik, S. V. Kravchenko, and T. M. Klapwijk, Phys. Rev. B 60, R5093 (1999).

[13] T. Okamoto, K. Hosoya, S. Kawaji, and A. Yagi, Phys. Rev. Lett. 82, 3875 (1999).

[14] $H_{\text {sat }}$ was chosen as the magnetic field where the resistivity has reached $90 \%$ of its constant high-field value.

[15] We thank Elihu Abrahams for this suggestion.

[16] An alternative explanation is that each Landau level splits into spin-up and spin-down sublevels in high magnetic field. It is unlikely that this would occur in every case at precisely the magnetic field where the resistance reaches saturation. Moreover, one expects the Landau levels to be broader for the larger resistances at high fields, making such splitting less likely. Also at $H>H_{\text {sat }}$ we do not see any interference effects corresponding to two spin bands.

[17] T. Ando, A. B. Fowler, and F. Stern, Rev. Mod. Phys. 54, 550 (1982).

[18] A. A. Abrikosov, Fundamentals of the Theory of Metals (North-Holland, Amsterdam, 1988).

[19] R. B. Dingle, Proc. R. Soc. London A 211, 517 (1952).

[20] A clear example can be found in Figs. 16 and 17 of D. R. Leadley et al., Phys. Rev. B 58, 13036 (1998). 